\title{
PIK3CA mutation in a case of CTNNB1- mutant sinonasal glomangiopericytoma
}

\author{
Christopher S. Hong, ${ }^{1}$ Mohammad Khan, ${ }^{2}$ Jordan M. Sukys, ${ }^{3}$ Manju Prasad, ${ }^{2}$ \\ E. Zeynep Erson-Omay, ${ }^{1}$ Eugenia M. Vining, ${ }^{3}$ and Sacit Bulent Omay ${ }^{1}$ \\ ${ }^{1}$ Department of Neurosurgery, ${ }^{2}$ Department of Pathology, ${ }^{3}$ Department of Surgery, Division of \\ Otolaryngology, Yale School of Medicine, New Haven, Connecticut 06510, USA
}

\begin{abstract}
Glomangiopericytomas are rare, primary sinonasal tumors. The existing literature is mostly limited to reports describing the clinicopathologic characteristics of these tumors. Comprehensive genetic characterization of glomangiopericytomas remains lacking. Wholeexome sequencing of a case of glomangiopericytoma was performed under an institutional review board-approved protocol. A 69-yr-old female underwent surgical resection of a glomangiopericytoma. Whole-exome sequencing revealed somatic mutations in CTNNB1 and PIK3CA, the former previously associated with this pathology but the latter not described. Concurrent dysregulation of $\mathrm{Wnt} / \beta$-catenin and PI3K/AKT/mTOR signaling, secondary to mutations in these two oncogenes, may be amenable to targeted treatment with existing clinically approved drugs. Genomic characterization of glomangiopericytomas remains lacking. This study reports novel coexistence of PIK3CA and CTNNB1 mutations in a case of glomangiopericytoma that may offer insight into the pathogenesis and potential for targeted medical therapies of this rare tumor.
\end{abstract}

[Supplemental material is available for this article.]

\section{INTRODUCTION}

Corresponding authors: emvining@mac.com; sacit.omay@yale.edu

(c) 2022 Hong et al. This article is distributed under the terms of the Creative Commons Attribution-NonCommercial

License, which permits reuse and redistribution, except for commercial purposes, provided that the original author and source are credited.

Ontology term: thick skull base

Published by Cold Spring Harbor Laboratory Press

doi:10.1101/mcs.a006120
Glomangioperictyomas, also known as sinonasal hemangioperictyomas, are rare mesenchymal neoplasms with a myoid phenotype, exhibiting positivity for smooth muscle actin (SMA), which distinguishes them from other soft tissue tumors (Park et al. 2017). They are rare tumors, comprising $<0.5 \%$ of all sinonasal tumors and considered to have low malignant potential (Stelow and Bishop 2017), but local recurrence and metastasis may occur in a subset of patients, despite complete surgical excision (Kazi et al. 2021). Most of the existing literature on glomangiopericytomas has been limited to single reports or case series, describing clinicopathologic characteristics. Comprehensive genetic study of glomangiopericytomas remains lacking, which may improve criteria for diagnosis and guide adjuvant treatment of surgically refractory tumors.

\section{RESULTS}

\section{Case Description}

A 69-yr-old female with no relevant past medical history, recent trauma, or carcinogenic exposures presented with recurrent epistaxis over the past year, increasing in frequency over the past several months. She underwent nasal endoscopy, which revealed a right nasal mass. Computed tomography (CT) demonstrated a hypodense, partially cystic mass in the 

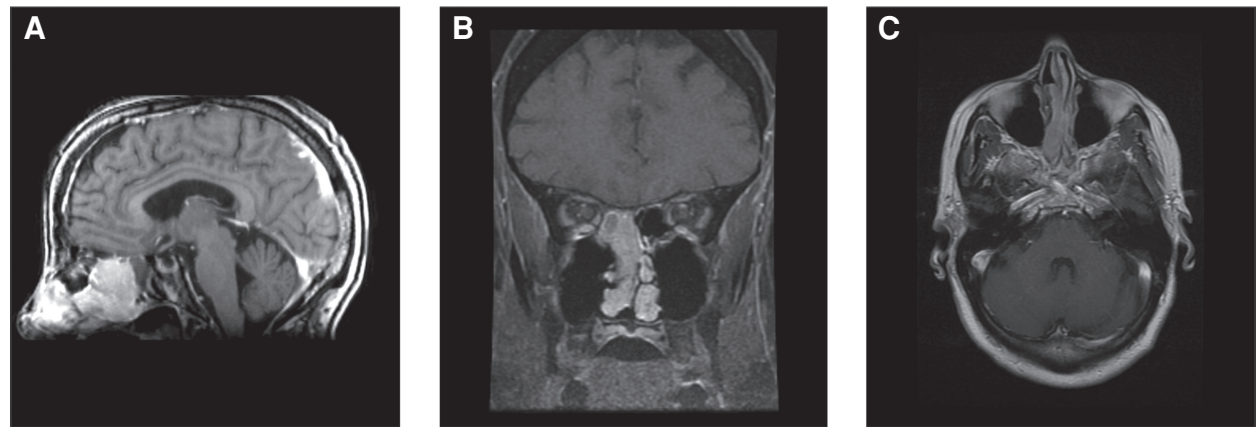

Figure 1. Representative $(A)$ sagittal, $(B)$ coronal, and $(C)$ axial slices of a T1-weighted magnetic resonance imaging (MRI) after gadolinium contrast demonstrate a $3.3 \times 1.2-\mathrm{cm}$ enhancing soft tissue mass within the right nasal cavity, protruding into the ipsilateral sphenoid sinus without definite intracranial extension.

right nasal cavity causing obstruction and opacification of the right posterior ethmoid air cells and sphenoid sinus, as well as bony remodeling of the ipsilateral cribriform plate and lateral lamella. Magnetic resonance imaging (MRI) demonstrated a $3.3 \times 1.2-\mathrm{cm}$ enhancing soft tissue mass, protruding into the ipsilateral sphenoid sinus without definite intracranial extension (Fig. 1). Subsequently, an endoscopic biopsy of the lesion revealed a diagnosis of sinonasal glomangiopericytoma, and after discussion at a multidisciplinary head and neck tumor board, local complete excision was recommended.

The patient subsequently underwent endoscopic endonasal resection of her tumor via a right total sphenoethmoidectomy, frontal sinusotomy, and maxillary antrostomy. Intra-operatively, the tumor was noted to attach medially on the superior posterior septum without extension through the septum and pushed the middle turbinate and superior turbinate laterally. There was no epidural involvement to suggest intracranial extension. Gross total resection was achieved with negative pathologic margins. The patient's postoperative course was uneventful, and further follow-up imaging is pending.

Pathologic review of the resected specimen revealed a tumor composed of bland spindle cells, arranged in sheets and ill-defined fascicles (Fig. 2A,B). There were tumor cells swirling around blood vessels showing perivascular hyalinization and focally formed a palisading pattern alternating with acellular hyalinized stroma. Focal mitoses was present (Ki-67 index 5\%-8\%) but without necrosis, atypical mitoses, or nuclear pleomorphism. Immunohistochemistry demonstrated diffusely positive staining for nuclear $\beta$-catenin and SMA (Fig. 2C,D). Stains for S100 and STAT6 were negative.

\section{Genomic Analysis}

Whole-exome sequencing (WES) revealed 19 somatic single-nucleotide variant (SNV)/indels with a variant allele frequency (VAF) $>10 \%$ in the tumor tissue. This translated into a relatively low tumor mutational burden (TMB) of 0.6 mut/Mb. We also analyzed the mutation signature for the missense mutations and identified $22 \%$ were $C: G>T: A, 5.6 \%$ were $C: G>A: T, 5.6 \%$ were $T: A>A: T, 39 \%$ were $T: A>C: G$, and $28 \%$ were $T: A>G: C$ alterations. This small number of missense mutations precluded assessment of a specific cancer-associated mutational signature (Alexandrov et al. 2013).

We prioritized the 19 somatic variants by the number of cases reported with the exact same mutation in the COSMIC database (Tate et al. 2019) and also with predicted pathogenicity, based on the ClinVar algorithm (Landrum et al. 2018). Based on these prioritizations, we identified two missense alterations, CTNNB1 (rs121913407, c.T133C: p.S45P) and PIK3CA (rs121913279, c.A3140G: p.H1047R) (Table 1). Both somatic variants have been 

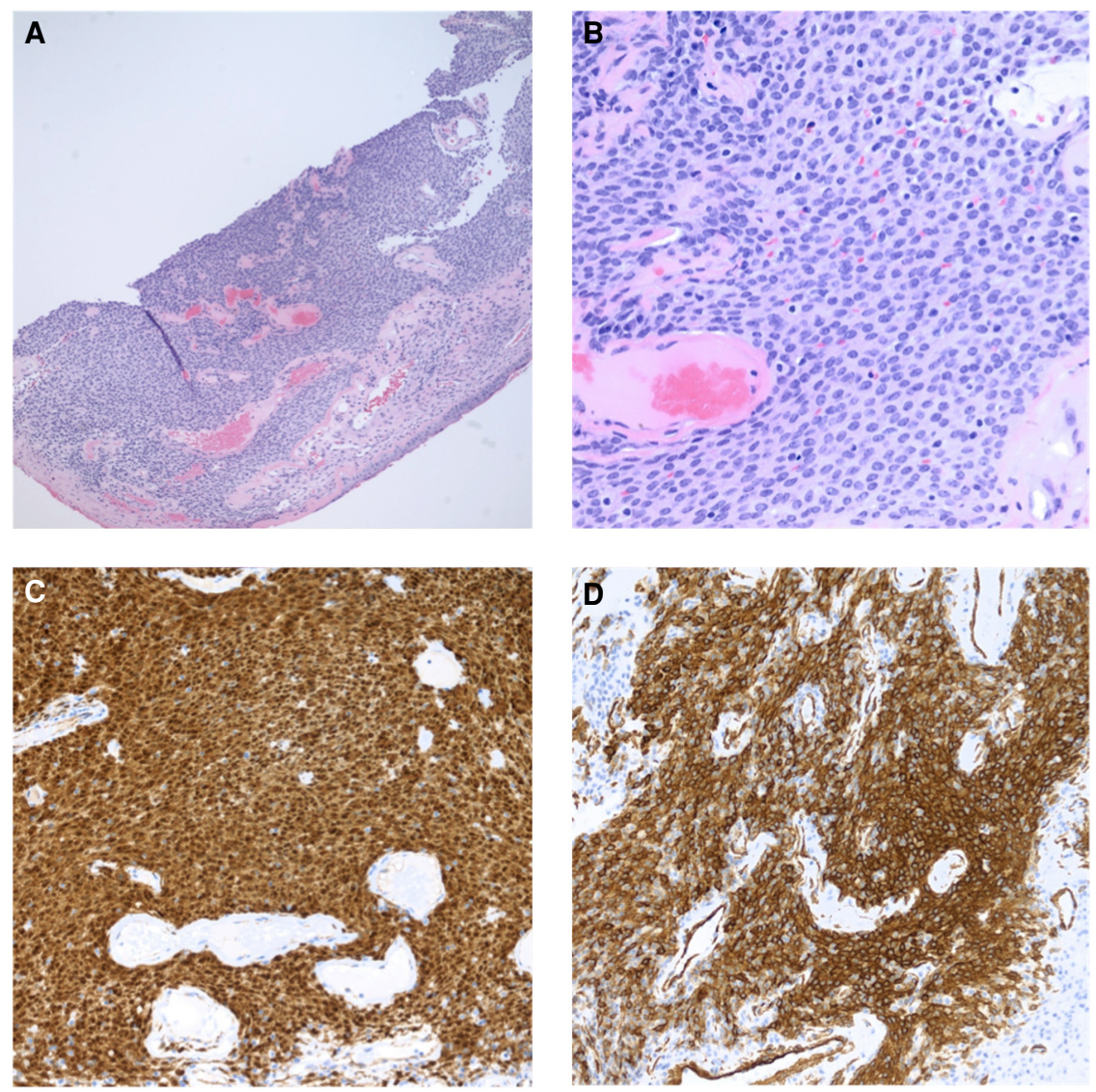

Figure 2. Histopathology. (A) A submucosal solid proliferating tumor with hyalinized vasculature is noted at low magnification (10x). (B) At higher power (40x), the tumor is comprised of monotonous ovoid to spindled cells. (C) The tumor cells are diffusely positive for $\beta$-catenin (nuclear) and (D) smooth muscle actin (SMA).

reported extensively in the COSMIC database (COSM5663 for CTNNB1, COSM775 for PIK3CA) and predicted to be highly pathogenic based on the SIFT (Vaser et al. 2016) and MutationTaster (Schwarz et al. 2014) algorithms. WES analysis did not identify any copy number or loss of heterozygosity events.

\section{DISCUSSION}

Glomangiopericytomas are a rare sinonasal tumor, now recognized as a subset of hemangiopericytoma arising from pericytes within the sinonasal region. Clinically, their diagnosis can be challenging to differentiate them from more common sinonasal tumors with malignant

Table 1. Genetic findings of index patient

\begin{tabular}{lccccccc}
\hline Gene & Chromosome & $\begin{array}{c}\text { HGVS DNA } \\
\text { reference }\end{array}$ & $\begin{array}{c}\text { HGVS protein } \\
\text { reference }\end{array}$ & $\begin{array}{c}\text { Variant } \\
\text { type }\end{array}$ & $\begin{array}{c}\text { Predicted } \\
\text { effect }\end{array}$ & dbSNP ID & $\begin{array}{c}\text { Genotype/variant allele } \\
\text { frequency }\end{array}$ \\
\hline CTNNB1 & 3 & c.T133C & p.S45P & Missense & Substitution & rs121913407 & NA/42.6\% \\
PIK3CA & 3 & c.A3140G & p.H1047R & Missense & Substitution & rs121913279 & NA/43.6\% \\
\hline
\end{tabular}


potential, such as various carcinomas and esthesioneuroblastoma, among others (Llorente et al. 2014). However, advances in molecular characterization of sinonasal tumors have aided in the ability to accurately differentiate these pathologies, such as the presence of NAB2STAT6 translocations in solitary fibrous tumors (Robinson et al. 2013) but otherwise absent in hemangiopericytomas (Agaimy et al. 2014). CTNNB1 mutations were subsequently shown to characterize glomangiopericytomas with positive immunohistochemical staining for $\beta$-catenin as a valid diagnostic marker (Haller et al. 2015; Lasota et al. 2015). In particular, the CTNNB1 p.S45F mutation, affecting the same protein sequence as in our patient, has demonstrated strong immunohistochemical nuclear localization as a means of pathway activation (Koike et al. 2019). However, further comprehensive genomic study of glomangiopericytoma remains lacking to guide diagnostic criteria and development of targeted therapies.

CTNNB1 mutations were originally associated with the pathogenesis of glomangiopericytomas by Haller et al. (2015). The authors performed WES in six patient samples and demonstrated recurrent hotspot CTNNB1 mutations in all cases, although notably lacking the NAB2-STAT6 fusion, characteristic of solitary fibrous tumors. Subsequently, several reports confirmed the prevalence of CTNNB1 mutations in glomangiopericytoma and their association with positive staining for $\beta$-catenin on immunohistochemistry (Lasota et al. 2015; Anzai et al. 2018; Kono et al. 2019; Obeidin et al. 2019). As such, $\beta$-catenin staining is now routinely used to clinically diagnose glomangiopericytomas and differentiate them from other soft tissue tumors of the sinonasal tract. The CTNNB1 variant (p.S45P) detected in our patient was also observed in one of the patients from the Haller et al. study and likewise has been reported as a pathogenic mutation in many systemic cancers according to the COSMIC database (Tate et al. 2019). Although there are no specific therapies targeting CTNNB1 directly, inhibition of tankyrase, a downstream regulator of Wnt/ $\beta$-catenin signaling, has demonstrated promising results in preclinical studies of $\beta$-catenin-driven colorectal cancers (Prossomariti et al. 2020). However, despite the prevalence of CTNNB1 mutations across the human cancer landscape, targeted therapies remain lacking, highlighting a need for further molecular characterization of glomangiopericytomas.

Our study notably revealed a PIK3CA mutation, a known oncogene in many solid human cancers (Samuels et al. 2004) but not previously reported in glomangiopericytoma. PI3Ks comprise a large group of kinases that regulate multiple downstream signaling pathways, affecting cellular proliferation, adhesion and motility, as well as survival. Activating mutations in the catalytic subunit P110a of PI3K (PIK3CA) leads to increased signaling through the canonical, oncogenic PI3K/Akt/mTOR pathway (Shayesteh et al. 1999). To our knowledge, this is the first report of a pathogenic PIK3CA mutation in glomangiopericytoma. Notably, there are several PI3K inhibitors in various stages of clinical development for advanced solid tumors, including alpelisib, buparlisib, copanlisib, and pictilisib (Vitale et al. 2021). Currently, alpelisib is clinically approved to treat patients with advanced PIK3CA-mutated breast cancer (André et al. 2021), whereas copanlisib is approved to treat relapsed lymphoma (Dreyling et al. 2017). Additionally, there are numerous clinically approved therapies targeting mTOR, a downstream effector of the PI3K/AKT pathway, most notably rapamycin and everolimus (Faivre et al. 2006). As such, there are existing clinically approved targeted therapies for $\mathrm{PI} 3 \mathrm{~K} / \mathrm{Akt} / \mathrm{mTOR}$ pathway dysregulated tumors, highlighting the need for further genomic characterization of glomangiopericytomas to determine whether targeting this pathway is a valid therapeutic strategy.

There are preclinical data to suggest that the $\mathrm{Wnt} / \beta$-catenin and PI3K/AKT/mTOR pathways work synergistically in promoting tumorigenesis (Solzak et al. 2017; Prossomariti et al. 2020). Interestingly, PIK3CA mutations may induce a senescent, stem cell-like state in HER2overexpressing breast tumors, which may together contribute to tumorigenesis (Chakrabarty et al. 2019). Potential for a similar synergistic relationship between PIK3CA and CTNNB1 mutations may exist and contribute to our understanding of glomangiopericytoma formation. 
Clinicopathologically, the presence of the PIK3CA mutation in our patient's tumor did not differentiate it significantly from other CTNNB1-mutated glomangiopericytomas in regard to measures, such as tumor size and Ki-67 index (Haller et al. 2015; Kono et al. 2019). As such, preclinical study of coexisting PIK3CA- and CTNNB1-mutated cancer models are needed to predict relevance for disease prognosis and management. Clinically, the combination of dysregulated Wnt/ $\beta$-catenin and PI3K/AKT/mTOR pathways has been best described in a subset of colorectal cancers (Prossomariti et al. 2020). Wnt/ $\beta$-catenin pathway dysregulated tumors may exhibit treatment resistance to targeted inhibition of the PI3K/AKT/mTOR pathway (Vitale et al. 2021). However, targeting both PIK3CA and mTOR simultaneously may be an effective therapeutic strategy in colorectal cancers driven by both PI3K and $\beta$-catenin signaling, characterized by PIK3CA and APC mutations (Foley et al. 2017). Although the prevalence of dysregulated $P I 3 K / A K T / m T O R$ signaling remains unclear in CTNNB1-mutated glomangiopericytomas, these aforementioned data suggest promising therapeutic potential in tumors, driven by overactivation of both oncogenic pathways.

This is the first report of the somatic genomic profile of glomangiopericytoma through WES, which revealed coexistence of CTNNB1 and PIK3CA mutations, presenting with a low tumor burden of 0.6 mut/Mb, similar to the burden reported in hemangiopericytomas (Robinson et al. 2013). Although CTNNB1 mutations have been associated with this pathology, PIK3CA mutations have not been reported until now. Notably, there appears to be a cross talk between the PI3K/Akt/mTOR and Wnt/ $\beta$-catenin signaling and therapeutic potential for dual inhibition of both pathways with existing clinically approved drugs. Further reports of comprehensive genetic analysis of glomangioperictyomas are needed to elucidate the prevalence of dysregulated PIK3CA activity in CTNNB1-mutated tumors.

\section{METHODS}

This study was conducted under an institutional review board-approved protocol at Yale University. The patient's blood and tumor tissue were collected after obtaining written informed consent. Histopathology, including immunohistochemical studies, was evaluated by a board-certified oncologic surgical pathologist.

WES and analysis was performed in accordance with our previously described methods at the Yale Cancer for Genome Analysis (YCGA) (Fomchenko et al. 2019). Briefly, genomic DNA from the tumor and blood were isolated and exome captured with IDT xGen Exome Research Panel v1 (Integrated DNA Technologies) and then sequenced on the Illumina NovaSeq6000 WES platform with $2 \times 100$ base pair reads. Downstream analysis of raw reads, including alignment, duplicate marking, realignment, and base quality recalibration was performed according to "GATK Best Practice" recommendations (v.4.1.9, Grch37). Somatic SNVs, indels, and copy-number variations (CNVs) were identified as previously described (Fomchenko et al. 2019). Mean coverage of $139.4 \times$ was achieved for blood and $240 \times$ for tumor tissue (Supplemental Table 1).

\section{ADDITIONAL INFORMATION}

\section{Data Deposition and Access}

All variants discussed in this manuscript have been submitted to the European Genome-phenome Archive (EGA: https://ega-archive.org/) under the accession ID EGAS00001005653. 


\section{Ethics Statement}

This study was conducted under an institutional review board-approved protocol at Yale University. The patient's blood and tumor tissue were collected after obtaining written informed consent.

\section{Author Contributions}

All authors were involved in the clinical care of the patient and drafting of the manuscript. C.S.H., E.Z.E.-O., and S.B.O. reviewed and conducted analysis of data from genomic sequencing.

\section{REFERENCES}

Agaimy A, Barthelmeß S, Geddert H, Boltze C, Moskalev EA, Koch M, Wiemann S, Hartmann A, Haller F. 2014. Phenotypical and molecular distinctness of sinonasal haemangiopericytoma compared to solitary fibrous tumour of the sinonasal tract. Histopathology 65: 667-673. doi:10.1111/his.12452

Alexandrov LB, Nik-Zainal S, Wedge DC, Aparicio SA, Behjati S, Biankin AV, Bignell GR, Bolli N, Borg A, Børresen-Dale AL, et al. 2013. Signatures of mutational processes in human cancer. Nature 500: 415421. doi:10.1038/nature12477

André F, Ciruelos EM, Juric D, Loibl S, Campone M, Mayer IA, Rubovszky G, Yamashita T, Kaufman B, Lu YS, et al. 2021. Alpelisib plus fulvestrant for PIK3CA-mutated, hormone receptor-positive, human epidermal growth factor receptor-2-negative advanced breast cancer: final overall survival results from SOLAR-1. Ann Oncol 32: 208-217. doi:10.1016/j.annonc.2020.11.011

Anzai T, Saito T, Tsuyama S, Toh M, Ikeda K, Ito S. 2018. A case of glomangiopericytoma at the nasal septum. Head Neck Pathol 12: 572-575. doi:10.1007/s12105-017-0870-6

Chakrabarty A, Surendran S, Bhola NE, Mishra VS, Wani TH, Baghel KS, Arteaga CL, Garg R, Chowdhury G. 2019. The H1047R PIK3CA oncogene induces a senescence-like state, pleiotropy and acute HSP90 dependency in HER2+ mammary epithelial cells. Carcinogenesis 40: 1179-1190. doi:10.1093/carcin/ bgz118

Dreyling M, Morschhauser F, Bouabdallah K, Bron D, Cunningham D, Assouline SE, Verhoef G, Linton K, Thieblemont C, Vitolo U, et al. 2017. Phase II study of copanlisib, a PI3K inhibitor, in relapsed or refractory, indolent or aggressive lymphoma. Ann Oncol 28: 2169-2178. doi:10.1093/annonc/mdx289

Faivre S, Kroemer G, Raymond E. 2006. Current development of mTOR inhibitors as anticancer agents. Nat Rev Drug Discov 5: 671-688. doi:10.1038/nrd2062

Foley TM, Payne SN, Pasch CA, Yueh AE, Van De Hey DR, Korkos DP, Clipson L, Maher ME, Matkowskyj KA, Newton MA, et al. 2017. Dual PI3K/mTOR inhibition in colorectal cancers with APC and PIK3CA mutations. Mol Cancer Res 15: 317-327. doi:10.1158/1541-7786.MCR-16-0256

Fomchenko El, Erson-Omay EZ, Zhao A, Bindra RS, Huttner A, Fulbright RK, Moliterno J. 2019. DNMT3A comutation in an IDH1-mutant glioblastoma. Cold Spring Harb Mol Case Stud 5: a004119. doi:10.1101/mcs .a004119

Haller F, Bieg M, Moskalev EA, Barthelme $\beta$ S, Geddert H, Boltze C, DiessI N, Braumandl K, Brors B, Iro H, et al. 2015. Recurrent mutations within the amino-terminal region of $\beta$-catenin are probable key molecular driver events in sinonasal hemangiopericytoma. Am J Pathol 185: 563-571. doi:10.1016/j.ajpath.2014.10.019

Kazi AA, McDougal EM, Howell JB, Schuman TA, Nord RS. 2021. Glomangiopericytoma: a case series with review of literature. Braz J Otorhinolaryngol doi:10.1016/j.bjorl.2021.02.007

Koike H, Nishida Y, Kohno K, Shimoyama Y, Motoi T, Hamada S, Kawai A, Ogose A, Ozaki T, Kunisada T, et al. 2019. Is immunohistochemical staining for $\beta$-catenin the definitive pathological diagnostic tool for desmoid-type fibromatosis? A multi-institutional study. Hum Pathol 84: 155-163. doi:10.1016/j.humpath 2018.09.018

Kono M, Bandoh N, Matsuoka R, Goto T, Akahane T, Kato Y, Nakano H, Yamaguchi T, Harabuchi Y, Nishihara H. 2019. Glomangiopericytoma of the nasal cavity with CTNNB1 p.S37C mutation: a case report and literature review. Head Neck Pathol 13: 298-303. doi:10.1007/s12105-018-0961-z

Landrum MJ, Lee JM, Benson M, Brown GR, Chao C, Chitipiralla S, Gu B, Hart J, Hoffman D, Jang W, et al. 2018. ClinVar: improving access to variant interpretations and supporting evidence. Nucleic Acids Res 46: D1062-D1067. doi:10.1093/nar/gkx1153

Lasota J, Felisiak-Golabek A, Aly FZ, Wang ZF, Thompson LD, Miettinen M. 2015. Nuclear expression and gain-of-function $\beta$-catenin mutation in glomangiopericytoma (sinonasal-type hemangiopericytoma) 
insight into pathogenesis and a diagnostic marker. Mod Pathol 28: 715-720. doi:10.1038/modpathol.2014 .161

Llorente JL, López F, Suárez C, Hermsen MA. 2014. Sinonasal carcinoma: clinical, pathological, genetic and therapeutic advances. Nat Rev Clin Oncol 11: 460-472. doi:10.1038/nrclinonc.2014.97

Obeidin F, Jennings LJ, Alexiev BA. 2019. Sinonasal glomangiopericytoma: a clinicopathologic study. Pathol Res Pract 215: 983-987. doi:10.1016/j.prp.2019.02.004

Park ES, Kim J, Jun SY. 2017. Characteristics and prognosis of glomangiopericytomas: a systematic review. Head Neck 39: 1897-1909. doi:10.1002/hed.24818

Prossomariti A, Piazzi G, Alquati C, Ricciardiello L. 2020. Are Wnt/ $\beta$-Catenin and PI3K/AKT/mTORC1 distinct pathways in colorectal cancer? Cell Mol Gastroenterol Hepatol 10: 491-506. doi:10.1016/j.jcmgh.2020.04 .007

Robinson DR, Wu YM, Kalyana-Sundaram S, Cao X, Lonigro RJ, Sung YS, Chen CL, Zhang L, Wang R, Su F, et al. 2013. Identification of recurrent NAB2-STAT6 gene fusions in solitary fibrous tumor by integrative sequencing. Nat Genet 45: 180-185. doi:10.1038/ng.2509

Samuels Y, Wang Z, Bardelli A, Silliman N, Ptak J, Szabo S, Yan H, Gazdar A, Powell SM, Riggins GJ, et al. 2004. High frequency of mutations of the PIK3CA gene in human cancers. Science 304: 554. doi:10.1126/science 1096502

Schwarz JM, Cooper DN, Schuelke M, Seelow D. 2014. MutationTaster2: mutation prediction for the deepsequencing age. Nat Methods 11: 361-362. doi:10.1038/nmeth.2890

Shayesteh L, Lu Y, Kuo WL, Baldocchi R, Godfrey T, Collins C, Pinkel D, Powell B, Mills GB, Gray JW. 1999. PIK3CA is implicated as an oncogene in ovarian cancer. Nat Genet 21: 99-102. doi:10.1038/5042

Solzak JP, Atale RV, Hancock BA, Sinn AL, Pollok KE, Jones DR, Radovich M. 2017. Dual PI3K and Wnt pathway inhibition is a synergistic combination against triple negative breast cancer. NPJ Breast Cancer 3: 17. doi:10.1038/s41523-017-0016-8

Stelow EB, Bishop JA. 2017. Update from the 4th edition of the World Health Organization classification of head and neck tumours: tumors of the nasal cavity, paranasal sinuses and skull base. Head Neck Pathol 11: 3-15. doi:10.1007/s12105-017-0791-4

Tate JG, Bamford S, Jubb HC, Sondka Z, Beare DM, Bindal N, Boutselakis H, Cole CG, Creatore C, Dawson E, et al. 2019. COSMIC: the Catalogue of Somatic Mutations in Cancer. Nucleic Acids Res 47: D941-D947. doi:10.1093/nar/gky1015

Vaser R, Adusumalli S, Leng SN, Sikic M, Ng PC. 2016. SIFT missense predictions for genomes. Nat Protoc 11: 1-9. doi:10.1038/nprot.2015.123

Vitale SR, Martorana F, Stella S, Motta G, Inzerilli N, Massimino M, Tirro E, Manzella L, Vigneri P. 2021. PI3K inhibition in breast cancer: identifying and overcoming different flavors of resistance. Crit Rev Oncol Hematol 162: 103334. doi:10.1016/j.critrevonc.2021.103334 


\section{COLD SPRING HARBOR Molecular Case Studies}

\section{PIK3CA mutation in a case of CTNNB1-mutant sinonasal glomangiopericytoma}

Christopher S. Hong, Mohammad Khan, Jordan M. Sukys, et al.

Cold Spring Harb Mol Case Stud 2022, 8: a006120 originally published online October 19, 2021 Access the most recent version at doi: $10.1101 /$ mcs.a006120
Supplementary http://molecularcasestudies.cshlp.org/content/suppl/2021/11/02/mcs.a006120.D Material C1
References This article cites 28 articles, 3 of which can be accessed free at: http://molecularcasestudies.cshlp.org/content/8/1/a006120.full.html\#ref-list-1
License This article is distributed under the terms of the Creative Commons Attribution-NonCommercial License, which permits reuse and redistribution, except for commercial purposes, provided that the original author and source are credited.
Email Alerting Receive free email alerts when new articles cite this article - sign up in the box at the Service top right corner of the article or click here.

